\title{
A new analysis tool for individual-level allele frequency for genomic studies
}

\author{
Hsin-Chou Yang ${ }^{1 *}$, Hsin-Chi Lin ${ }^{1}$, Mei-Chu Huang ${ }^{1}$, Ling-Hui Li ${ }^{2}$, Wen-Harn Pan², Jer-Yuarn Wu' ${ }^{2}$, Yuan-Tsong Chen ${ }^{2}$
}

\begin{abstract}
Background: Allele frequency is one of the most important population indices and has been broadly applied to genetic/genomic studies. Estimation of allele frequency using genotypes is convenient but may lose data information and be sensitive to genotyping errors.

Results: This study utilizes a unified intensity-measuring approach to estimating individual-level allele frequencies for 1,104 and 1,270 samples genotyped with the single-nucleotide-polymorphism arrays of the Affymetrix Human Mapping $100 \mathrm{~K}$ and $500 \mathrm{~K}$ Sets, respectively. Allele frequencies of all samples are estimated and adjusted by coefficients of preferential amplification/hybridization (CPA), and large ethnicity-specific and cross-ethnicity databases of CPA and allele frequency are established. The results show that using the CPA significantly improves the accuracy of allele frequency estimates; moreover, this paramount factor is insensitive to the time of data acquisition, effect of laboratory site, type of gene chip, and phenotypic status. Based on accurate allele frequency estimates, analytic methods based on individual-level allele frequencies are developed and successfully applied to discover genomic patterns of allele frequencies, detect chromosomal abnormalities, classify sample groups, identify outlier samples, and estimate the purity of tumor samples. The methods are packaged into a new analysis tool, ALOHA (Allele-frequency/Loss-of-heterozygosity/Allele-imbalance).

Conclusions: This is the first time that these important genetic/genomic applications have been simultaneously conducted by the analyses of individual-level allele frequencies estimated by a unified intensity-measuring approach. We expect that additional practical applications for allele frequency analysis will be found. The developed databases and tools provide useful resources for human genome analysis via high-throughput singlenucleotide-polymorphism arrays. The ALOHA software was written in R and R GUI and can be downloaded at http://www.stat.sinica.edu.tw/hsinchou/genetics/aloha/ALOHA.htm.
\end{abstract}

\section{Background}

Allele frequency denotes the relative frequency of an allele compared with the total frequency of all alleles at a marker locus. It is one of the most important population indices and has been broadly applied to genetic/ genomic research [1-5]. The generalized concept of allele frequency has two aspects: individual-level allele frequency and population-level allele frequency. The former represents a within-individual relative frequency of alleles and its standard error reflects inter-cell variability in an individual; the latter represents a within-population relative frequency of alleles and its standard error reflects inter-individual variability in a population [6].

\footnotetext{
* Correspondence: hsinchou@stat.sinica.edu.tw

${ }^{1}$ Institute of Statistical Science, Academia Sinica, Taipei 115, Taiwan
}

Using the most abundant genetic marker in the human genome, namely the single-nucleotide polymorphism (SNP), both individual-level and populationlevel allele frequencies can be estimated using a (genotype-based) allele-counting approach and an (intensitybased) intensity-measuring approach (Appendix A). This paper focuses on the intensity-measuring allele frequency because this allele frequency estimate is insensitivity to genotyping errors and preserves data information that might be lost in conventional genotype-based analyses. For instance, the genomic abnormality of a particular sample, for instance NA18996 from the Japanese in population in the International HapMap Project [7-10], can be easily observed by individual-level allele frequencies using an intensity-measuring approach [Additional file 1, Supplemental Figure S1 (A)] but not 
by using an allele-counting approach [Additional file 1, Supplemental Figure S1 (B)]. The finding motivated us to analyze intensity-based allele frequencies for extraction of information lost in conventional genotype-based analyses.

An intensity-measuring approach can accurately estimate allele frequencies with the aid of an adjustment for preferential amplification/hybridization [11]. The coefficient of preferential amplification/hybridization (CPA) is used to quantify preferential amplification/hybridization and reduce the estimation bias of allele frequency [12-19]. Consider a SNP with genotype $A A, A a$ or $a a$. In comparison with an unadjusted individual-level allele frequency [Additional file 2, Supplemental Figure S2 (A)], a CPA-adjusted frequency of allele $A$ moves toward the expected values of 1, 0.5 and 0 for SNPs with genotypes $A A, A a$ and $a a$, respectively [Additional file 2, Supplemental Figure S2 (B)]. In comparison with an unadjusted population-level allele frequency from a DNA pool of 240 individuals [Additional file 3, Supplemental Figure S3 (A)], a CPA-adjusted frequency of allele $A$ moves toward the true allele frequencies obtained from an individual genotyping experiment [Additional file 3, Supplemental Figure S3 (B)] [6]. The results demonstrate the important role of CPA adjustment in allele frequency estimation, thereby expanding applications of allele frequency in various genomic science disciplines. The features of CPA have been studied, but not exhaustively $[14,15,17]$. Therefore, we undertook an in-depth investigation of the relationship between CPA and important factors including sample size, time of data acquisition, effects of laboratory site, type of gene chip, ethnicity effects, and phenotypic status, and we constructed new public CPA and allele frequency databases.

Population-level allele frequency has many important applications and has been well discussed [1-4]. This paper mainly focuses on individual-level allele frequency and investigates its applications in genetic/genomic studies, including the discovery of allele frequency patterns, identification of chromosomal aberrations (including aneuploidy, loss of heterozygosity $(\mathrm{LOH})$, and allelic imbalance (AI)), outlier detection, and sample classification (including stratification by population and gender). This is the first time that these important genetic/genomic applications have been simultaneously conducted based on individual-level allele frequencies estimated by a unified intensity-measuring approach.

\section{Methods}

\section{Samples}

This study analyzes genotype and intensity data from several large genomic projects. The first dataset consists of 367 and 448 Taiwanese samples genotyped using the
Affymetrix Human Mapping 100K Set and 500K Set (Affymetrix, San Diego, CA, USA), respectively, from the Taiwan Han Chinese Cell and Genome Bank [20]. The second dataset consists of 175 and 198 hypertension patients genotyped using the Affymetrix Human Mapping 100K Set and 500K Set, respectively, from the Academia Sinica Multi-Centered Hypertension Genetic Study. The third dataset consists of 30 African trios of Yoruba in Ibadan (YRI), 30 Caucasian trios of residents in CEPH Utah (CEU), and 90 Asians (45 Han Chinese in Beijing (CHB) and 45 Japanese in Tokyo (JPT)) from the International HapMap Project [7-10], where all 270 samples were genotyped using both the Affymetrix Human Mapping 100K Set and 500K Set. The fourth dataset consists of 242 and 304 leukaemia cancer patients who were genotyped using the Affymetrix Human Mapping 100K Set and 500K Set, respectively; in addition, 50 normal controls were genotyped with the Affymetrix Human Mapping $100 \mathrm{~K}$ and $500 \mathrm{~K}$ Sets from an acute lymphoblastic leukaemia (ALL) project $[21,22]$. All participants involved in the genomic projects signed Informed Consent Forms.

\section{Genotyping}

All samples were genotyped with the Affymetrix Human Mapping 100K Set and/or 500K Set (Affymetrix, San Diego, CA, USA), which contain 116,204 SNPs with a median inter-marker distance of $8.5 \mathrm{~kb}$ and 500,568 SNPs with a median inter-marker distance of $2.5 \mathrm{~kb}$, respectively. The data sheets and genotyping manuals for the two SNP chips can be downloaded at http:// www.affymetrix.com/. For details of the SNP genotyping experiments refer to Yang et al. [23] for the Taiwanese normal samples and hypertensive patients, The International HapMap Consortium [7-10] for the International HapMap Project samples, and Mullighan et al. [21,22] for the ALL Project samples. SNP genotype calling algorithms, DM (Dynamic Model) [24] and BRLMM (Bayesian Robust Linear Model with Mahalanobis Distance Classifier) [25], were used for the Affymetrix Human Mapping 100K Set and 500K Set, respectively.

\section{Allele frequency estimation}

An individual-level allele frequency is the proportion of a specific allele in a genotype. Here we formulate the existing procedures of allele frequency estimation. Consider an example of a SNP with genotype $a a, A a$ or $A A$. Two methods can be used to estimate an individuallevel allele frequency. First, the allele-counting approach uses genotype data from an individual genotyping experiment (IGE). The number of allele $A$ is counted and then used to calculate the proportion of allele $A$ in a genotype. Besides no calls, there are only three possible outcomes of an allele frequency estimate for a SNP. 
Therefore, allele frequency estimates are $0,0.5$ and 1, corresponding to genotypes $a a, A a$ and $A A$, respectively (Equation (A1)). Second, the intensity-measuring approach uses intensity data from an IGE. The frequency of allele $A$ is estimated by calculating the ratio of intensities pertaining to allele $A$ relative to the total intensity of two alleles, where intensities are adjusted by considering a CPA. The ratio reflects a relative amount of allele $A$ compared with the total amount of two alleles at a SNP for an individual (Equation (A2)).

In contrast to an individual-level allele frequency, a population-level allele frequency is the proportion of a specific allele in a study population. The allele frequencies can be estimated based on data from an IGE or a pooled allelotyping experiment (PAE). In an IGE, individual-level allele frequencies are estimated by using genotype data or intensity data as mentioned above. Then a population-level allele frequency is estimated by taking an average over individual-level allele frequencies from genotype data (Equation (A3)) or intensity data (Equation (A4)). In a PAE, intensity data are available, but individual genotype data are not. A relative intensity of allele $A$ in a DNA pool, which is constructed by mixing genomic DNA from multiple samples, is calculated to estimate a population-level allele frequency (Equation (A5)). The detailed procedures for allele frequency estimation and CPA adjustment are described in Appendix A.

\section{Identification of chromosomal aberrations}

We develop multiple chromosomal aberration indices and a sliding-window approach in concert with a standard individual-level allele frequency plot to identify chromosomal aberrations such as aneuploidy, AI, LOH, long-contiguous-stretch-of-homozygosity (LCSH), and so on. For an individual and a SNP, individual-level allele frequency is compared with genotype-specific reference confidence intervals. Index $A_{i, m}$ is used to detect AI genomic segments characterized by SNP points with allele frequencies outside the allele frequency confidence intervals of three genotypes. Index $L_{i, m}$ is used to detect $\mathrm{LOH}$ or $\mathrm{LCSH}$, genomic regions characterized by SNP points with contiguous homozygous calls (Appendix B). The two indices are used to identify unusual SNPs point by point. Furthermore, based on all patients and normal controls, two multipoint indices, $A_{i, m}^{*}$ (Equation (B1)) and $L_{i, m}^{*}$ (Equation (B2)) are calculated in each window using a sliding-window approach. In addition, the smoothed indices, $\tilde{A}_{i, m}^{*}(w)$ and $\tilde{L}_{i, m}^{*}(w)$, are also calculated using a spline smoothing technique. We identify chromosomal aberrations by pinpointing the genomic regions where the indices of a patient are higher than the 95\%-quantile indices of normal controls. Detailed procedures for identifying chromosomal aberrations are described in Appendix B.

\section{Sample classification and outlier detection}

We apply an allele frequency biplot based on individuallevel allele frequencies to classify samples and detect outliers. An allele frequency biplot, which uses a singular value decomposition to decompose an allele frequency matrix into a sample matrix and a SNP matrix (Equations (C1) and (C2)), projects samples and SNPs onto a two-dimensional plane simultaneously. The first dimension is a sample coordinate constructed by the first two columns of the sample matrix, and the second dimension is a SNP coordinate constructed by the first two columns of the SNP matrix. The rank-2 biplot configuration can be utilized for sample classification, outlier detection and SNP clustering. First, sample classification allows for samples with a similar allele frequency distribution to be clustered and used to study population stratification and gender grouping. Second, outlier detection identifies samples far away from the majority of samples for a further examination prior to downstream analyses. Third, SNP clustering identifies a collection of SNPs physically close to specific sample groups. The SNP patterns are used to separate sample groups and explain sample characteristics. Detailed procedures for constructing an allele frequency biplot are described in Appendix C.

\section{Results}

Coefficient of preferential amplification/hybridization

We characterize genomic patterns of CPA based on the three genome projects, the Taiwan Han Chinese Cell and Genome Bank [20], the Academia Sinica Multi-Center Hypertension Genetic Study, and the International HapMap Project [7-10]. Both Affymetrix 100K and $500 \mathrm{~K}$ Sets are considered. This work extends our previous study by using richer study samples and denser SNP chips [17]. We calculate CPA based on an unbiased estimator [12] and construct public CPA databases for the Taiwanese population, ethnic-specific populations (African, Asian, and Caucasian populations), and a cross-ethnicity group (a combination of African, Asian and Caucasian populations). The CPA databases are available online at http://140.109.72.48/index.htm. Three CPA query methods (keyword query, general query and advanced query) are provided. In addition, we examine the relationship between CPA and important factors including: (1) sample size $(45,90,180$, and 367 individuals); (2) time of data acquisition (four genotyping periods) and effects of laboratory site (two sites); (3) gene chip type (Affymetrix Human Mapping 100K Set and 500K Set); (4) ethnicity effects (Africans, Asians, and Caucasians); and (5) phenotypic status (normal controls 
and young-onset hypertension patients). Results of the investigation of these issues are summarized in order they are presented here.

First, CPA can be estimated well for a moderate sample size [Additional file 4, Supplemental Figure S4 (A)], but its variance decreases significantly with increasing sample size [Additional file 4, Supplemental Figure S4 (B)]. Based on a total of 367 Taiwanese samples that were genotyped with the Affymetrix Human Mapping 100K Set, 45, 90 and 180 samples are randomly selected from the 367 samples. Pairwise correlation coefficients of $\log _{2}(\mathrm{CPA})$ obtained from different sample sizes are calculated, and the lowest correlation coefficient is greater than 0.975 . In the fitted quadratic regression, an intercept term is close to 0 (i.e., the regression curve passes through the origin), and the regression coefficients of the linear term and quadratic term are close to 1 and 0 , respectively. In other words, CPAs are close even when they are calculated from a relatively small sample size, implying that CPA can be estimated well even for a moderate sample size [Additional file 4, Supplemental Figure S4 (A)]. Note that, with respect to each SNP, only heterozygous individuals have been used to estimate CPA. The expected number of heterozygous individuals is the number of total samples multiplied by the probability that a SNP is heterozygous. CPA variances pertaining to the four sample sizes are calculated. The overall SNP averages of ratios of CPA variances of 180 versus 367 samples (green points), 90 versus 367 samples (red points), and 45 versus 367 samples (blue points) are 1.42, 1.99, and 3.90, respectively. The larger the sample size, the smaller the variability of CPA [Additional file 4, Supplemental Figure S4 (B)].

Second, CPA is insensitive to data from different genotyping time periods [Additional file 5, Supplemental Figure S5 (A)] and different laboratories [Additional file 5, Supplemental Figure S5 (B)]. To evaluate time effect on CPA, CPAs are calculated based on data from the same genotyping laboratory but from four time periods of data acquisition (2005/05/04, 2006/01/09, 2006/03/17 and 2006/06/29), and sample sizes in experiments are close (95, 96, 90 and 76). All the pairwise correlation coefficients of $\log _{2}(\mathrm{CPA})$ are greater than 0.966 , and quadratic regression curves act like a linear regression line passing through the origin and having a slope of 1 , implying that CPA is robust to data from different genotyping periods [Additional file 5, Supplemental Figure S5 (A)]. We also examine the effect of genotyping sites on CPA. CPAs are calculated based on data from the 90 Asian samples in the International HapMap Project and 95 Taiwanese samples. These two datasets with similar sample sizes were collected from Asian populations but genotyped at different times and sites. A high correlation of CPA between the two datasets is found across chromosomes, implying that CPA is robust to data from different genotyping sites [Additional file 5, Supplemental Figure S5 (B)].

Third, CPAs from the Affymetrix Human Mapping $100 \mathrm{~K}$ and $500 \mathrm{~K}$ Sets yield similar genomic patterns [Additional file 6, Supplemental Figures S6 (A) - (D)]. Means (standard deviations) of $\log _{2}(\mathrm{CPA})$ for $100 \mathrm{~K}$ and 500K Sets are $0.04(0.54)$ and 0.05 (0.70), respectively. The majority of $\log _{2}(\mathrm{CPA})$ are bounded by \pm 1 . Moreover, we examine genome-wide distributions of CPA for different ethnic groups (CHB, JPT, YRI, and CEU), Taiwanese samples and their combination) and different SNP chips (Affymetrix $100 \mathrm{~K}$ and $500 \mathrm{~K}$ Sets). The results show that genome-wide CPA can be well modeled by using log-normal distributions, where the results of Taiwanese samples are shown [Additional file 7, Supplemental Figures S7 (A) and (B)]. The finding is useful for discussions of theoretical sampling distribution of CPA-adjusted allele frequency estimates and statistical tests.

Fourth, genomic distributions of CPA between ethnic groups may differ [Additional file 8, Supplemental Figures S8 (A) and (B)]. CPAs are calculated based on data from different populations, including $45 \mathrm{Han}$ Chinese (CHB), 45 Japanese (JPT), 60 Africans (YRI), 60 Caucasians (CEU), 90 Asians $(\mathrm{CHB}+\mathrm{JPT})$, and $210 \mathrm{com}-$ bined samples $(\mathrm{CHB}+\mathrm{JPT}+\mathrm{YRI}+\mathrm{CEU})$. Results show that correlation of CPAs among populations is dependent on the ethnic populations selected [Additional file 8, Supplemental Figure S8 (A)]. Among all pair-wise comparisons, two Asian populations, $\mathrm{CHB}$ and JPT, have the highest correlation $(r=0.966)$, depicted by a flat ellipse; the two Asian populations and Caucasian populations have the lowest correlation ( $\mathrm{r}=0.845$ for $\mathrm{CHB}$ and $\mathrm{CEU}$ and $r=0.845$ for JPT and CEU), depicted by round ellipses. Note that comparison of the Caucasian and the combined Asian populations present a nephroid ellipse, where the $95 \%$ confidence interval of the mean regression curve does not pass through the origin, and the slope term deviates from unity. This suggests a relatively large discrepancy of CPAs between Asian and Caucasian populations. CPAs of the African population are located in an intermediate position between the Asian and Caucasian populations, and the order is shown in a tree diagram [Additional file 8, Supplemental Figure S8 (B)].

Finally, CPA distributions between hypertension patients and normal controls are similar [Additional file 9, Supplemental Figure S9]. CPAs are calculated based on a total of 180 Taiwanese normal control samples and 175 Taiwanese hypertension patient samples that were genotyped with the Affymetrix Human Mapping 100K Set in the same laboratory. A high correlation $(r=$ 0.980 ) is observed. This result implies that the pooled 
DNA association mapping of a Taiwanese hypertension study may use the same CPA for case and control groups.

\section{Allele frequency}

Based on our CPA databases, we estimate individuallevel allele frequencies using the intensity-measuring approach for samples from different populations, including (1) Affymetrix Human Mapping 100K Set: 367 Taiwanese samples and 270 HapMap samples, and (2) Affymetrix Human Mapping 500K Set: 448 Taiwanese samples and 270 HapMap samples. The pattern of allele frequency can be observed in a standard allele frequency plot. For example, for a normal female sample (NA19206) from the YRI population in the International HapMap Project (The International HapMap Consortium, 2003-2007), with the exception of some noisy SNP points, an allele frequency plot exhibits three bands formed by SNPs with an individual-level allele frequency close to 0, 0.5 or 1 (Figure 1 and Equation (A2)). Allele frequency plots of cancer patients with chromosomal aberrations will be shown later in Identification of Chromosomal Aberrations. In addition, we establish individual-level allele frequency databases that provide SNP annotation, allele frequencies and the summary statistics for the HapMap samples for both the Affymetrix Human Mapping $100 \mathrm{~K}$ and $500 \mathrm{~K}$ Sets. The databases are available online at http://140.109.72.48/index.htm.
Three allele frequency query methods (keyword query, general query and advanced query) are provided. The individual-level allele frequency estimates and databases are applied to genomic pattern detection, chromosomal aberration analysis, classification analysis, and outlier detection.

\section{Identification of chromosomal aberrations}

Individual-level allele frequency can be used to identify chromosomal aberrations, such as aneuploidy, LOH, AI, and so on, which contribute to the complex genomic profiles of many cancer patients. Based on our constructed databases of CPA and individual-level allele frequency, we apply the developed sliding-window $\mathrm{LOH}$ and AI detectors to identify chromosomal aberrations for the 242 and 304 ALL patients that were genotyped with the Affymetrix Human Mapping 100K Set and 500K Set, respectively, in the ALL Project. Two examples are given here to demonstrate whole-chromosome aberration and segment aberration. The first example is a male leukaemia patient with hyperdiploidy (Hyperdip50-SNP-\#27). His genome exhibits AI on chromosomes $6,8,10,14,17$, and 18 and $\mathrm{LOH}$ on chromosomes 2, 19 and 22 (Figure 2 and Appendix B). The second example is a male T-cell ALL patient ( $\mathrm{T}$ ALL-SNP-\#49). Three chromosomal regions, 2p, 6q, and $9 \mathrm{p}$, of AI are identified, with chromosomes $6 \mathrm{q}$ and $9 \mathrm{p}$ also show LOH (Figure 3 and Appendix B).

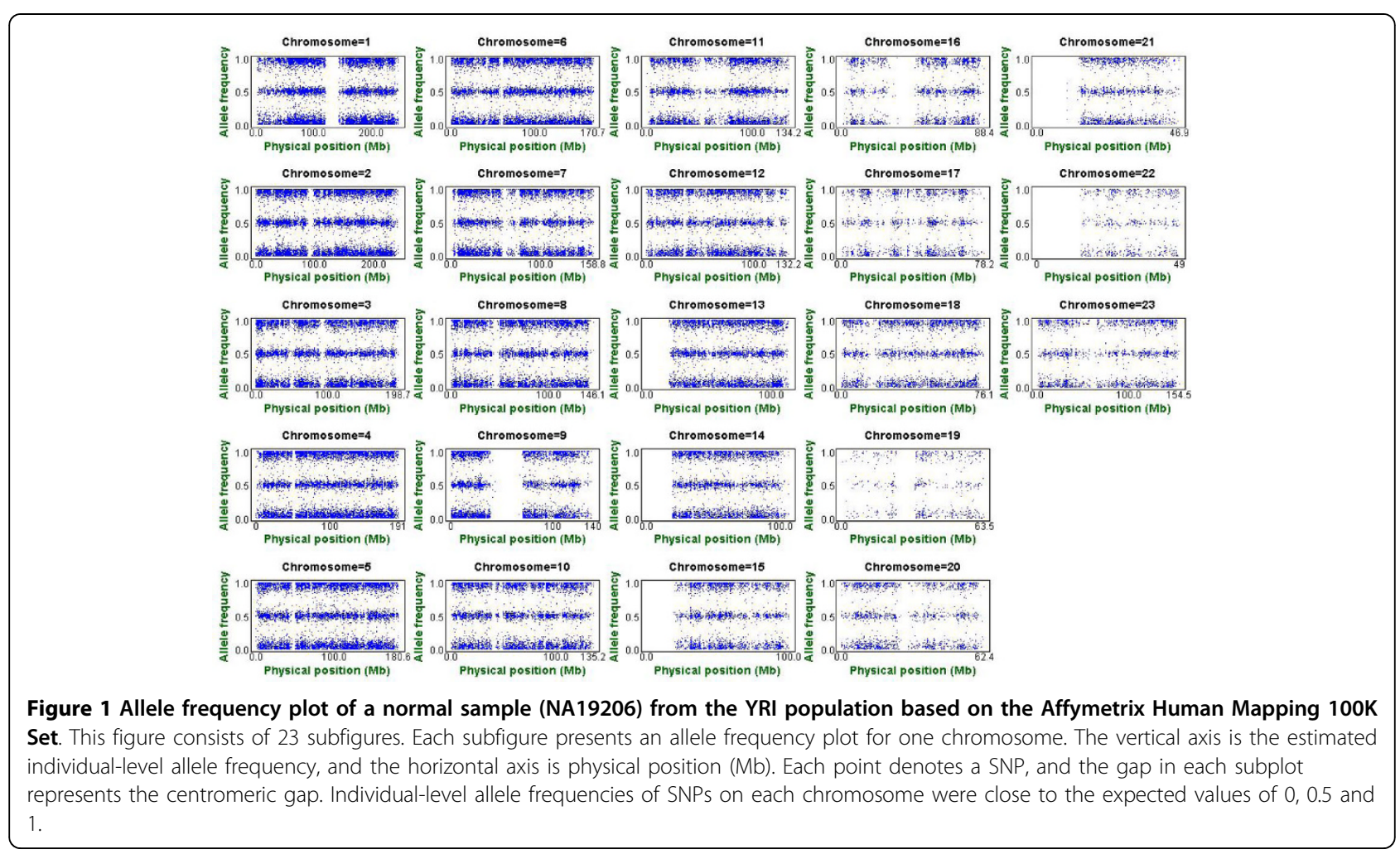



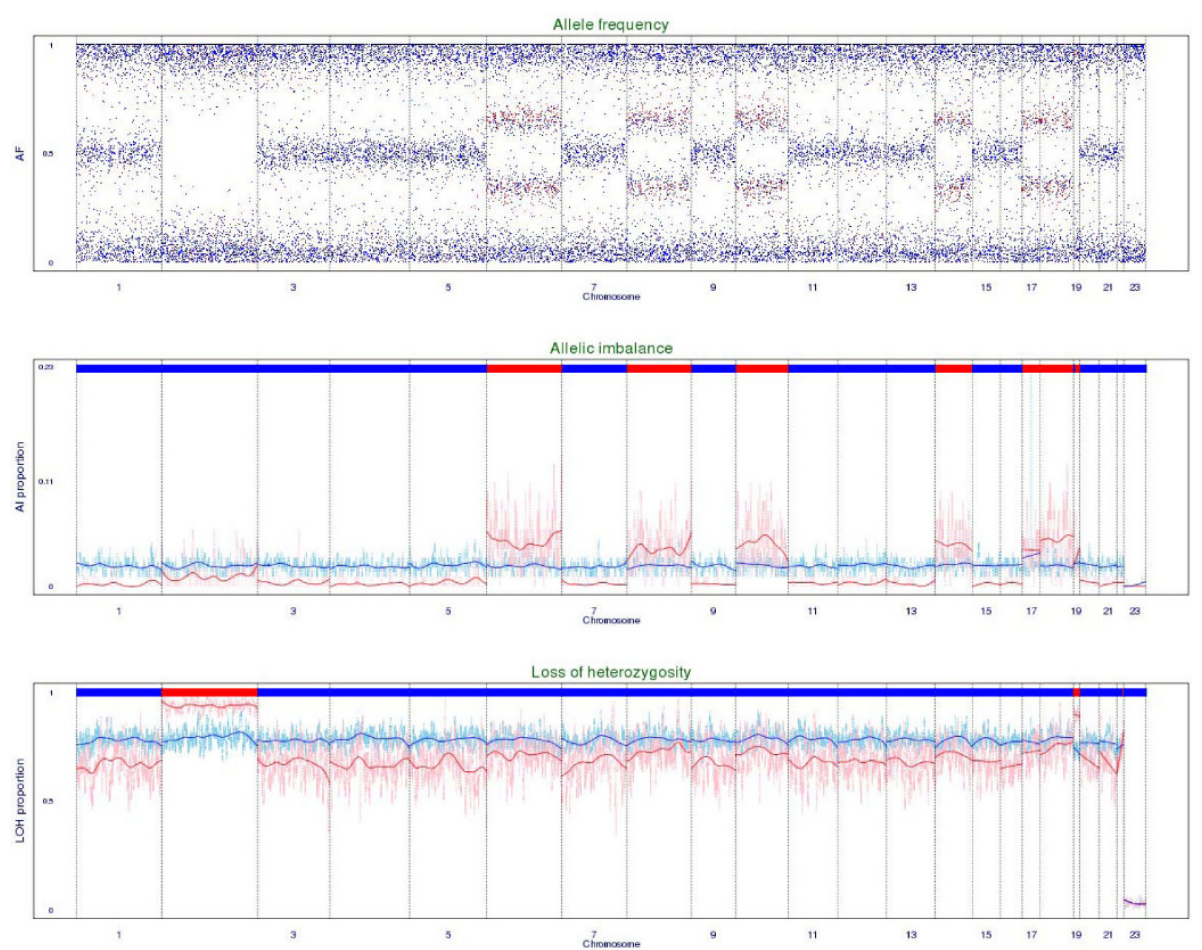

Figure 2 Chromosomal aberration plots of a hyperdiploidy leukaemia patient based on the Affymetrix Human Mapping 100K Set. A chromosomal aberration plot consists of an extended allele frequency plot, an allelic imbalance (Al) plot, and a loss of heterozygosity (LOH) plot from the top to the bottom (Appendix B). In the extended allele frequency plot, a lot of the Al SNPs (red points) were found on whole chromosomes 6, 8, 10, 14, 17, 18 and 19. In the Al plot, a whole-chromosome region of Al (red bar in the top panel) was found on chromosomes $6,8,10,14,17,18$ and 19. In the LOH plot, a whole-chromosome region of LOH (red bar in the top panel) was found on chromosomes 2 and 19 .

High proportions of $\mathrm{LOH}$ and $\mathrm{AI}$ are found in the ALL study. In the analysis of 242 patients genotyped with the Affymetrix $100 \mathrm{~K}$ Set, 215 samples (88.80\%) with AI are found, and 93 of these (43.26\%) have wholechromosome AI. Among the 242 patients, 154 samples $(63.63 \%)$ with $\mathrm{LOH}$ are found, and 26 of these (16.88\%) have whole-chromosome LOH. In the analysis of 304 patients genotyped with the Affymetrix 500K Set, 261 samples (85.86\%) with AI are found, and 53 of these (20.31\%) have whole-chromosome AI. Among the 304 patients, 240 samples (78.95\%) with $\mathrm{LOH}$ are found, and 35 of these (14.58\%) have whole-chromosome LOH.

\section{Group classification and outlier detection}

Based on individual-level allele frequencies, an allele frequency biplot is developed to classify samples belonging to different ethnic groups and gender groups and identify outlier samples.

First, in an unsupervised cluster analysis of the International HapMap Project data, the majority of samples from African (YRI), Caucasian (CEU) and Asian (CHB and JPT) populations are clearly classified into three groups in an allele frequency biplot (Figure 4 and
Appendix C). Furthermore, samples from Chinese (CHB) and Japanese (JPT) populations are also accurately classified as two distinct groups (Figure 5 and Appendix C). The results illustrate that individual-level allele frequencies are not only useful to distinguish the genetic differences of the Asian, Caucasian and African populations, but also able to distinguish the small differences between Asian subpopulations.

Second, allele frequencies can be used to judge the gender of a person from which a sample was obtained. Genetically, a female has two X chromosomes and a male has only one $\mathrm{X}$ chromosome. High homozygosity on the $\mathrm{X}$ chromosome should generally be observed in a male sample [Additional file 2, Supplemental Figure S2 (B) for a male and Figure 1 for a female]. Our analysis shows that an allele frequency biplot for the $\mathrm{X}$ chromosome can classify the gender of samples donors [Additional file 10, Supplemental Figure S10].

Third, allele frequency biplots can be used to detect outliers. For example, a JPT sample (NA1902) is some distance away from all other samples in a biplot of chromosome 2 (Figure 5) because this sample has a LCSH on this chromosome. 

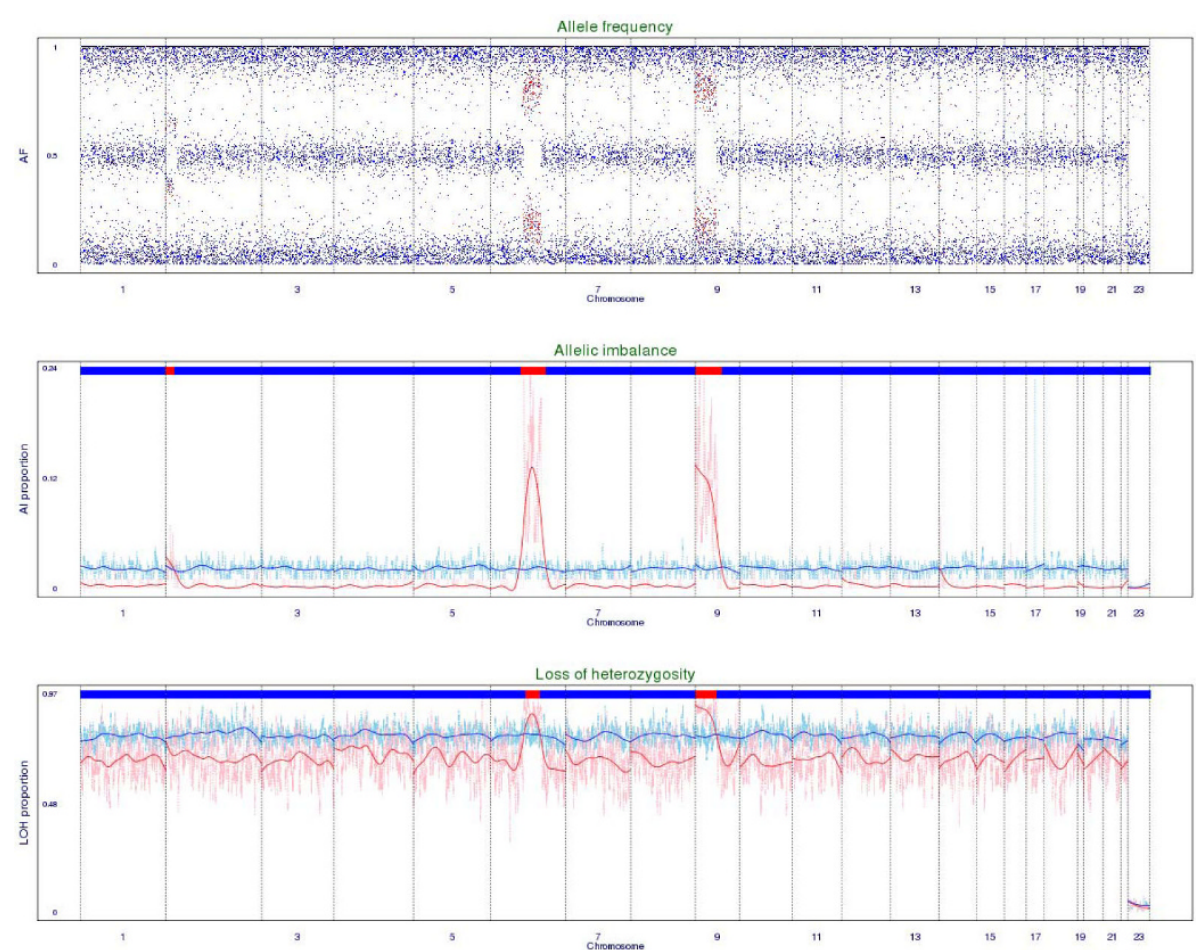

Figure 3 Chromosomal aberration plots of a T-ALL patient based on the Affymetrix Human Mapping 100K Set. A chromosomal aberration plot consists of an extended allele frequency plot, an allelic imbalance (Al) plot, and a loss-of-heterozygosity (LOH) plot from the top to the bottom (Appendix B). In the extended allele frequency plot, clusters of AI SNPs (red points) were found on chromosomal regions of $2 p$, $6 q$ and $9 p$. The regions of $2 p, 6 q$ and $9 p$ were also identified in the Al plot (red bar in the top panel). The regions of $6 q$ and $9 p$ were also identified in the LOH plot (red bar in the top panel).
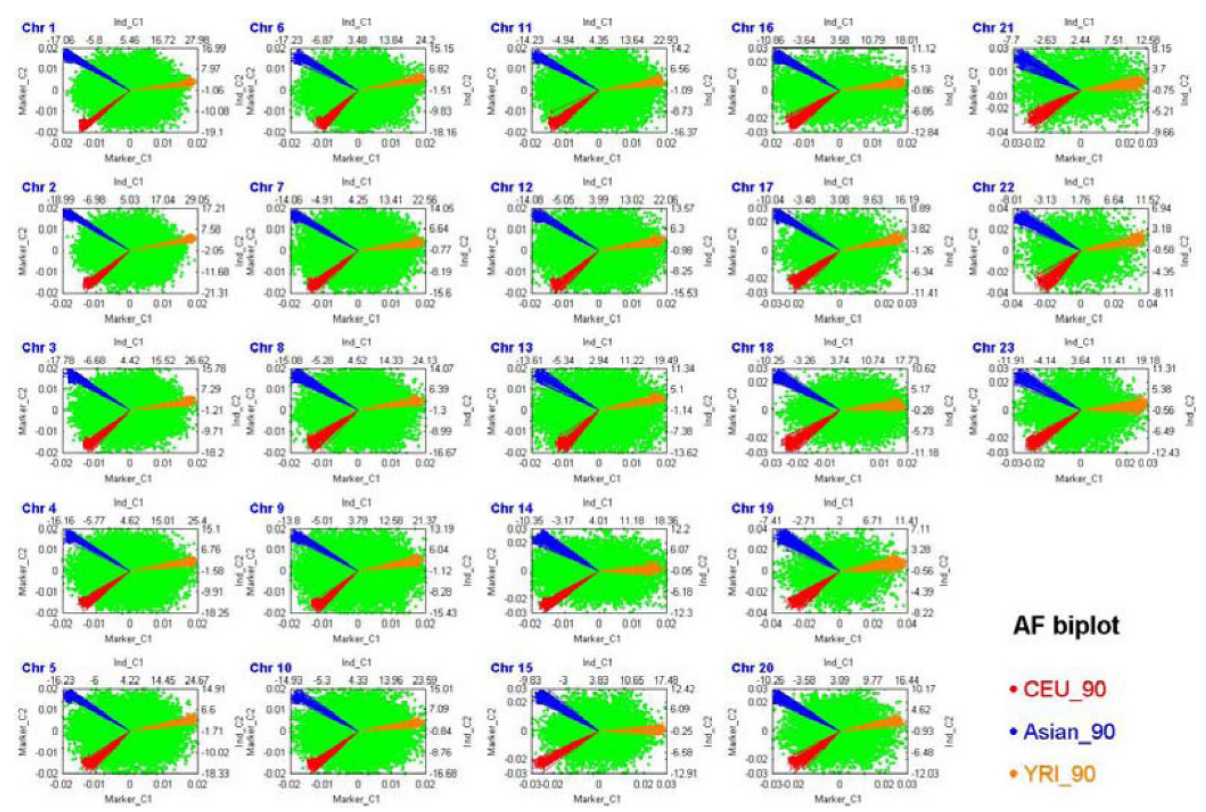

AF biplot

- CEU_90

- Asian_90

-YRI_90

Figure 4 Allele frequency biplots of the three populations in the International HapMap Project dataset based on the Affymetrix Human Mapping 500K Set. A genome-wide allele frequency biplot of data for 90 CEU, 90 YRI, and 90 subjects of Asian descent (45 CHB and $45 \mathrm{JPT}$ ). The figure consists of 23 subfigures. Each subfigure presents an allele frequency biplot of one chromosome, where the four axes reflect the first two individual components and the first two SNP components in a rank-2 biplot (Appendix C). Samples from YRI (yellow line), CEU (red line) and Asian (blue line) were clearly classified into three groups. 

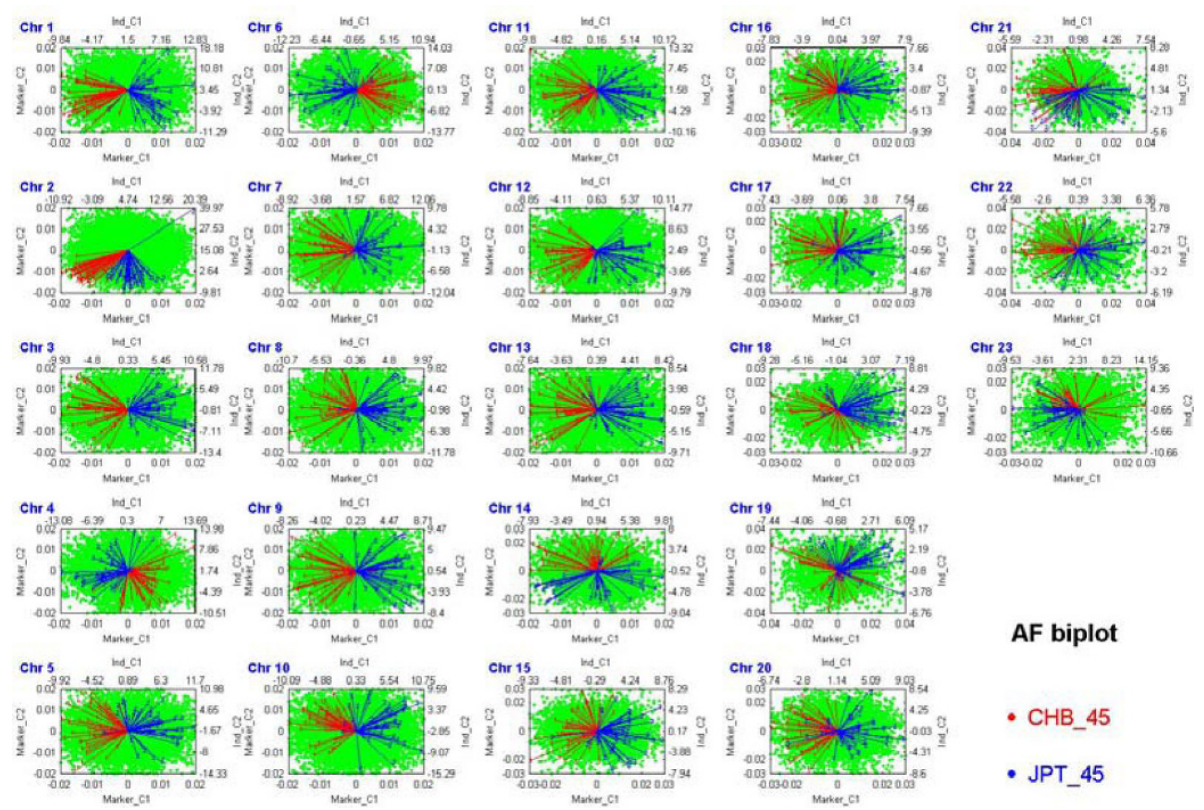

Figure 5 Allele frequency biplots of the Asian population in the International HapMap Project dataset based on the Affymetrix Human Mapping 500K Set. A genome-wide allele frequency biplot of data for 90 subjects of Asian descent (45 CHB and 45 JPT). The figure consists of 23 subfigures. Each subfigure presents an allele frequency biplot of one chromosome, where the four axes reflect the first two individual components and the first two SNP components in a rank-2 biplot (Appendix C). Samples from CHB (red line) and JPT (blue line) are accurately classified as two distinct groups.

\section{Discussion}

We apply individual-level allele frequencies, estimated using an intensity-measuring approach, to genetic and genomic studies. Under the framework, statistical inferences are drawn based on intensity data instead of genotype data. Compared with a genotype-based analysis that estimates allele frequencies via an allele-counting approach, our method has three advantages. First, the method can analyze data from DNA samples reflecting clonal heterogeneity and containing DNA from contaminating "normal" cells, which is often the case in cancer studies. Second, the method can be used to analyze data from mixed DNA of multiple samples for cost savings. Third, the method is insensitive to genotype calling errors, which frequently occur in aberrant chromosomal regions, such as segments with aneuploidy, LOH, AI, and so on. The performance of the methods developed for amplification/hybridization calibration, allele frequency estimation, group classification, outlier detection, and genomic aberration identification are examined comprehensively based on the data from large-scale genomic projects.

The proposed methods are not restricted to the Affymetrix GeneChip, but also are adapted to the Illumina BeadChip. For example, the individual-level allele frequency plot of a normal sample genotyped with the Illumina HumanHap550-Duo BeadChip also yields the expected three-band pattern similar to what is obtained with the Affymetrix GeneChip [Additional file 11, Supplemental Figure S11]. In addition to applications for individual-level allele frequency, the proposed intensitymeasuring approach can be easily applied to study population-level allele frequency. For example, in a pooled allelotyping experiment [2,26-31], individual genotype information is not available, thereby the allelecounting approach fails to be applied; the proposed intensity-measuring approach, however, works well in this type of experiment (Appendix A). Allele frequency estimates of the pooled allelotyping experiment of a pool size of 240 are quite accurate compared to the true answers obtained from the individual genotyping experiment of all 240 samples $(r=0.983)$ [Additional file 3, Supplemental Figure S3 (B)]. Pooled DNA is constructed by mixing DNA from multiple individuals; therefore, allelotyping of pooled DNA provides a costsaving alternative to an individual genotyping experiment and has been recognized as an efficient tool for identification of polymorphisms and mutations, homozygosity mapping, genetic association, and so on.

We have constructed large ethnicity-specific and cross-ethnicity CPA databases. Compared with our previous database [17], the new databases are improved by increased sample size and the addition of a dense SNP chip platform. Researchers may use our free database, 
thereby curtailing study costs. The characteristics of CPA extracted from our databases are summarized as follows. First, CPA variance decreases significantly as sample size increases; thus, a CPA database should be based on a large number of samples. Second, CPA is independent of the time and laboratory where the genotyping took place, and therefore the CPAs provided in our databases are applicable to studies carried out at different times and places. Third, the $100 \mathrm{~K}$ and $500 \mathrm{~K}$ gene chips yield similar CPA patterns. Fourth, discrepancies of CPAs in ethnic groups discourage the direct use of CPA from different ethnic groups. Fifth, most SNPs have a strongly positively correlated genomic distribution of CPA between phenotypic groups of hypertension. Sixth, log-normal distributions capture the genomic distributions of CPA well, and CPA is associated with GC content and genotype. These findings broaden our understanding of preferential amplification/ hybridization and will benefit a wide variety of genetic/ genomic studies. In addition, we also constructed a database of individual-level allele frequency, which provides useful materials for genetic/genomic research discussed in this paper.

Interestingly, when compared to the result of DNA copy number changes in T-ALL-SNP-\#49 reported by Mullighan et al. [21], AI of chromosome 2p correlates well with amplification, whereas AI and LOH of chromosomes $6 \mathrm{q}$ and $9 \mathrm{p}$ are the result of deletions. These results demonstrate the feasibility of applying individuallevel allele frequencies to identify chromosomal aberrations. Our method can be jointly used with some of the existing copy number analysis tools such as LB [32], PennCNV [33], QuantiSNP [34], dCHIP [35], and GenoCN [36] to move toward a more complete understanding of the mechanisms underlying chromosomal abnormalities.

In addition to identification of chromosomal aberrations, individual-level allele frequency can also be used to identify an occurrence of cell mixture. JPT sample NA18996 is an example with "abnormal" genomic patterns [Additional file 1, Supplemental Figure S1 (A)] that obviously deviate from the three-band pattern of a normal sample [Additional file 2, Supplemental Figure S2 (B)]. The individual-level allele frequency plot of sample NA18996 shows that the majority of this individual's genome have five allele frequency bands with the exception that some autosomal regions have two or three bands and the $\mathrm{X}$ chromosome has four bands, indicating that this sample may reflect a mixture of DNA samples. In addition, we show that individual-level allele frequency can also be used to estimate the level of contaminating "normal" cells (Appendix D). In the example of T-ALLSNP-\#49, the average allele frequencies $\left(f_{1}\right)$ of the abnormal regions of $6 \mathrm{q}$ and $9 \mathrm{p}$ were as follows: $f_{1}=0.192$ for
$6 \mathrm{q}$, and $f_{1}=0.190$ for $9 \mathrm{p}$, implying an estimated range of contaminating "normal" cells of $0.235-0.250$, which is close to the reported sample purity (blast percentage $=$ 76\%) reported by Mullighan et al. [21].

Our analyses show that individual-level allele frequencies and allele frequency biplots are useful for distinguishing genetic differences among ethnic populations. On the one hand, the method can be applied to examine population stratification, which is one of the most common factors underlying false positives in case-control association studies, through the identification of subgroups. On the other hand, the method is potentially useful to assign ethnicity for individuals with unclear ancestry information by superimposing a new sample point to the established ethnic groups using an allele frequency biplot, or to assign unknown forensic individuals to pre-study groups. Compared with a principal component analysis that can also be applied to study sample classification, an allele frequency biplot provides a more detailed visual appraisal of the relationship between SNPs and samples (Appendix C).

\section{Conclusion}

Allele frequency has been studied for many years. With the aid of whole-genome SNP chip technology, highthroughput SNP data have become available. Based on the individual-level allele frequencies obtained from the unified estimation procedure, we developed new methods/software/databases to extract hidden genetic information and broaden the potential application of allele frequency to genomic research. Our new analysis tool, ALOHA (Allele-frequency/Loss-of-heterozygosity/ Allele-imbalance), written in R and R GUI, provides for genome-wide analysis of allele frequency and detection of both LOH and AI. An allelefrequency biplot is also provided for sample classification, outlier detection, and SNP clustering. The software can be downloaded at http://www.stat.sinica.edu.tw/hsinchou/genetics/aloha/ ALOHA.htm. New allele frequency and CPA databases have been created. Functions for data query modes (keyword query, general query, and advanced query) and data downloads are provided. The databases are available at http://140.109.72.48/index.htm. We expect that additional practical applications of allele frequency will be found and allele frequency analysis will continue to play a key role in genetic/genomic research.

\section{Appendices}

\section{Appendix A. Allele frequency estimation}

We summarize the estimation procedures for two types of allele frequencies, individual-level and populationlevel allele frequency, in this appendix. This summary focuses on the most abundant genetic marker, the SNP, in the human genome. SNP data can be generated from 
two types of SNP typing experiments, namely an individual genotyping experiment (IGE) and a pooled allelotyping experiment (PAE), which can be carried out using a customized platform (e.g., MALDI-TOF mass spectrometry) or a genome-wide platform (e.g., Affymetrix and Illumina SNP chips).

An IGE collects intensity data (e.g., mass spectrometry peak intensity provided by MALDI-TOF mass spectrometry or hybridization intensity provided by Affymetrix SNP chips) and then uses a SNP calling algorithm to assign genotype calls to SNPs based on intensity data. Both individual-level and population-level allele frequencies can be estimated by using an allele-counting approach based on genotypic data and an intensity-measuring approach based on intensity data.

In contrast to an IGE, which analyzes DNA from each individual, a PAE analyzes mixed DNA from multiple individuals. With PAE it is difficult to extract genotype information for individuals although the same genotyping platform is employed for PAE as IGE. A PAE collects intensity data but cannot determine individual genotypes, and is therefore unable to estimate individual-level allele frequency; nevertheless, a PAE provides a cost-efficient way to estimate population-level allele frequency by using an intensity-measuring approach.

We introduce the estimating procedures as follows. Suppose that there are $n$ independent individuals in total. Consider a SNP with genotype $A A, A a$ or $a a$. Let $\left(p_{i}, i=1, \ldots, n\right)$ denote individual-level allele frequencies for $n$ individuals and $p$ denote a population-level allele frequency of allele $A$. In an IGE, we collect two types of data for each individual: (1) genotypes $\left(G_{i}, i=1, \ldots, n\right)$, and (2) pairs of intensities of two alleles $\left\{\left(S_{1 i}, S_{2 i}\right), i=1, \ldots\right.$, $n\}$. In a PAE, we collect a pair of intensities of two alleles $\left(S_{1}, S_{2}\right)$ in a DNA pool with a pool size of $n$.

First, we introduce the estimating procedures for an individual-level allele frequency, which is a proportion of a specific allele in a genotype. An individual-level allele frequency can be estimated using an allele-counting approach based on genotypic data and an intensity-measuring approach based on intensity data from an IGE.

The allele-counting approach uses genotype data from an IGE. The number of allele $A$ is counted and then used to calculate the proportion of allele $A$ in a genotype. Under the assumptions of Hardy-Weinberg equilibrium and random sampling, the formulae of the estimator of allele frequency of allele $A$ and its expectation and variance can be written as follows:

$$
\hat{p}_{i}=I\left[G_{i}=A A\right]+\frac{I\left[G_{i}=A a\right]}{2}, E\left(\hat{p}_{i-}=p_{i} \text { and } V\left(\hat{p}_{i}\right)=\frac{p_{i}\left(1-p_{i}\right)}{2},\right.
$$

where $I[E]$ is an indicator taking a value of 1 if event $E$ holds; otherwise, the value is 0 .
The intensity-measuring approach uses "adjusted" intensity data from an IGE, where intensities are "adjusted" by considering a coefficient of preferential amplification/hybridization (CPA). Based on intensity data from individuals who are heterozygous at the SNP, CPA can be estimated as follows $[6,12,17]$ :

$$
\hat{\kappa} \mathrm{U}=\frac{1}{n_{\text {heter }}} \sum_{j=1}^{n_{\text {heter }}} \frac{S_{1 i}}{S_{2 i}}+\frac{n_{\text {heter }}}{n_{\text {heter }}-1}\left(\frac{\bar{S}_{1}}{\bar{S}_{2}}-\frac{1}{n_{\text {heter }}} \sum_{j=1}^{n_{\text {heter }}} \frac{S_{1 i}}{S_{2 i}}\right),
$$

where $\left(\bar{S}_{1}, \bar{S}_{2}\right)$ are the sample means of intensities of individuals and $n_{\text {heter }}$ is the number of individual heterozygous for the SNP. For each individual, the frequency of allele $A$ is estimated by calculating the ratio of adjusted intensities pertaining to allele $A$ relative to the total intensity of the two alleles. The estimator and its expectation and variance can be written as follows:

$$
\tilde{p}_{i}=\frac{S_{1 i}}{S_{1 i}+\hat{\kappa}_{U} \cdot S_{2 i}}, E\left(\tilde{p}_{i}\right) \approx p_{i} \text { and } V\left(\tilde{p}_{i}\right) \approx \frac{p_{i}\left(1-p_{i}\right)}{2}+p_{i}^{2}\left(1-p_{i}\right)^{2} \gamma_{\hat{\kappa} U}^{2}+\sigma^{2},
$$

where a first-order Taylor expansion is used to calculate of the approximate expectation and variance, $\gamma_{\hat{\kappa}_{U}}^{2}$ is the coefficient of variation of CPA, and $\sigma^{2}$ is the experimental variation.

Second, we introduce procedures to estimate a population-level allele frequency, which is a proportion of a specific allele in a study population. The allele frequencies can be estimated based on data from an IGE or a PAE. In an IGE, a population-level allele frequency can be estimated by taking an average over individual-level allele frequencies from genotype data (Equation (A1)) or intensity data (Equation (A2)). The estimators and their expectations and variances can be written as follows:

$$
\begin{aligned}
& \hat{p}=\sum_{i=1}^{n} \hat{p}_{i} / n, E(\hat{p})=p \text { and } V(\hat{p})=\frac{p(19 P)}{2 n} \\
& \tilde{p}=\sum_{i=1}^{n} \tilde{p}_{i} / n, E(\tilde{p}) \approx p \text { and } V(\tilde{p}) \approx \frac{1}{n} \sum_{i=1}^{n}\left[\frac{p_{i}\left(1-p_{i}\right)}{2}\right]+\frac{1}{n} \sum_{i=1}^{n} p_{i}^{2}\left(1-p_{i}\right)^{2} \gamma_{\hat{K}_{U}}^{2}+\sigma^{2} .
\end{aligned}
$$

In a PAE, relative intensities of allele $A$ in a DNA pool, which is constructed by mixing genomic DNA from $n$ samples, are calculated to estimate a populationlevel allele frequency. The estimator and its expectation and variance can be written as follows:

$$
\tilde{f}=\frac{S_{i}}{S_{1}+\hat{\kappa}_{\mathrm{U}} \cdot S_{2}}, E(\tilde{f}) \approx p \text { and } V(\tilde{f}) \approx \frac{p(1-p)}{2 n}+p^{2}(1-p)^{2} \gamma_{\hat{\kappa}_{U}}^{2}+\sigma^{2} \text {.(A5) }
$$

Appendix B. Identification of chromosomal aberrations A sliding-window approach, aided by individual-level allele frequency plots, is developed to identify chromosomal aberrations. All SNPs are arranged in order of 
physical position on a study chromosome. Let $G_{i, m}$ and $\tilde{f}_{i, m}$ denote the genotype data and the individual-level allele frequency of the $m$ th SNP of the $i$ th SNP chip. For any SNP $m$, the genotype-specific reference mean and standard deviation of the individual-level allele frequency can be calculated based on a large number of reference samples as follows:

$$
\hat{\mu}_{G_{i m}}=\frac{\sum_{i=1}^{n} \tilde{f}_{i, m} \cdot I\left[G_{i, m}=G_{m}\right]}{\sum_{i=1}^{n} I\left[G_{i, m}=G_{m}\right]} \text { and } \hat{\sigma}_{G_{i m}}=\frac{\left.\sum_{i=1}^{n}\left\{\left(\tilde{f}_{i, m}-\hat{\mu}_{G_{m}}\right)\right\} \cdot I\left[G_{i, m}=G_{m}\right]\right\}^{2}}{\sum_{i=1}^{n} I\left[G_{i, m}=G_{m}\right]}
$$

where $G_{m}$ is $A A, A a$ or $a a$. Then three genotype-specific reference confidence intervals can be written as follows:

$$
R=\left\{R_{G_{m}}=\left[\hat{\mu}_{G_{m}}+Z_{\alpha / 2} \hat{\sigma}_{G_{m}}, \hat{\mu}_{G_{m}}+Z_{\alpha / 2} \hat{\sigma}_{G_{m}}\right], \quad G_{m}=(A A, A a, a a)\right\} .
$$

The first single-point index $A_{i, m}=I\left[\tilde{f}_{i, m} \notin R\right]$ is used to detect an allelic-imbalance (AI) SNP with allele frequency outside the allele frequency confidence intervals of the three genotypes. The second single-point index $L_{i, m}=I\left[\tilde{f}_{i, m} \notin R_{A a}\right]$ is used to detect a loss-of-heterozygosity (LOH) or long-contiguous-stretch-of-homozygosity (LCSH) SNP with allele frequency outside the allele frequency confidence intervals of a heterozygous call. In other words, SNP $m$ of individual $i$ is identified as an AI SNP point if $A_{i, m}=1$ and identified as an $\mathrm{LOH} / \mathrm{LCSH}$ SNP point if $L_{i, m}=1$. Two multilocus sliding-window chromosomal aberration detectors are developed as follows. The AI index can be written as

$$
A_{i, m}^{*}(w)=\sum_{j \in \mathfrak{I}_{m}(w)} A_{i, j} / \#\left(\mathfrak{I}_{m}(w)\right)
$$

and the $\mathrm{LOH} / \mathrm{LCSH}$ index as

$$
L_{i, m}^{*}(w)=\sum_{j \in \mathfrak{I}_{m}(w)} L_{i, j} / \#\left(\mathfrak{I}_{m}(w)\right)
$$

where

$\mathfrak{I}_{m}(w)=\{\max \{1, m-w\}, \Lambda, m-1, m, m+1, \Lambda, \min \{m+w, M\}\}$ denotes a study window with an anchor SNP $m$ and $\#\left(\mathfrak{I}_{m}(w)\right)$ denotes the window size, i.e., the number of SNPs within the window. The indices are calculated for all normal controls and patients. We calculate a 95\%quantile of normal samples for index $A_{i, m_{\tilde{A}}^{*}}^{*}(w)$ and for index $L_{i, m}^{*}(w)$. The smoothed indices, $\tilde{A}_{i, m}^{*}(w)$ and $\tilde{L}_{i, m}^{*}(w)$, are calculated using a spline smoothing or local fit technique, such as a penalized regression [37]. We identify unusual genomic regions where the indices of a patient are higher than the $95 \%$-quantile indices of normal controls in the human genome. The methods are useful for quickly scanning the human genome and accurately determining the starting and ending positions of chromosomal aberration regions for every patient.
A chromosomal aberration plot consisting of three components is used to detect special genomic patterns such as aneuploidy, $\mathrm{LOH}$, and AI (Figures 2 and 3). The first component is an extended allele frequency plot where AI SNPs $\left(A_{i, m}=1\right)$ are depicted by red points and non-AI SNPs $\left(A_{i, m}=0\right)$ are depicted by blue points. The second component is an AI plot where index $A_{i, m}^{*}(w)$ of a patient and the $95 \%$ quantile of $A_{i, m}^{*}(w)$ of normal samples are displayed as light-red points and light-blue points, respectively, and smoothed versions are displayed as deep-red points and deep-blue points, respectively. The top panel shows a red bar that denotes an AI region if $\tilde{A}_{i, m}^{*}(w)$ is greater than the $95 \%$ quantile of $\tilde{A}_{i, m}^{*}(w)$ of normal samples; otherwise, a blue bar is shown. The third component is a $\mathrm{LOH}$ plot where index $L_{i, m}^{*}(w)$ of a patient and the 95\% quantile of $L_{i, m}^{*}(w)$ of normal samples are displayed at light-red points and light-blue points, respectively, and smoothed versions are displayed as deep-red and deep-blue points, respectively. The top panel shows a red bar that denotes an $\mathrm{LOH}$ region if $\tilde{L}_{i, m}^{*}(w)$ is greater than the $95 \%$ quantile of $\tilde{L}_{i, m}^{*}(w)$ of normal samples; otherwise, a blue bar is shown.

\section{Appendix C. Allele frequency biplot}

The biplot invented by Gabriel [38] was applied to explore microarray gene expression data $[39,40]$ and LOH [41]. This paper first applies a biplot to visualize individual-level allele frequency data for hundreds of thousands of SNPs and large samples. Let A denote the individual-level allele frequency matrix with $R$ rows (SNPs) and $C$ columns (samples). Let $\mathbf{F}=\mathbf{A}-(1 / C) \cdot \mathbf{A} \cdot \mathbf{1}$, where 1 is a $C \times 1$ column vector with cell values of 1 . The matrix $\mathbf{F}$ is decomposed into a multiplication of an $R \times K$ SNP-effect matrix (row-effect matrix) $\mathbf{R}$ and a $C \times K$ sample-effect matrix (column-effect matrix) C using a singular value decomposition as outlined below. First, allele frequency matrix $\mathbf{F}$ is decomposed by a singular value decomposition as follows:

$$
\mathbf{F}=\mathrm{PDQ} \text {. }
$$

$\mathbf{P}$ is an $R \times R$ orthogonal matrix (i.e., $\mathbf{P} \mathbf{P}^{\prime}=\mathbf{I}_{R}$ ), and the columns of $\mathbf{P}$ are eigenvectors of $\mathbf{F F} ; \mathbf{Q}$ is an $R \quad C \quad$ orthogonal matrix (i.e., $\mathbf{Q Q}^{\prime}=\mathbf{I}_{R}$ and $\mathbf{Q Q}^{\prime}=\mathbf{I}_{C}$ ) and the columns of $\mathbf{Q}$ are eigenvectors of $\mathbf{F}^{\prime} \mathbf{F}$; $\mathbf{D}$ is an $R \times R$ diagonal matrix having $w$ nonnegative elements as the square root of nonnegative eigenvalues of FF' and F'F, where $w=R \quad C$. Second, allele frequency matrix $\mathbf{F}$ is further partitioned into row-effect and column-effect matrices as follows:

$$
\mathbf{F}=\mathbf{R C} \text {, }
$$


where $\mathbf{R}=\mathbf{P}$ and $\mathbf{C}=\mathbf{D Q}$ for a SNP-effect biplot, and $\mathbf{R}=\mathbf{P D}$ and $\mathbf{C}=\mathbf{Q}$ for a sample-effect biplot. Note that $\mathbf{F F}^{\prime}=\mathbf{R} \mathbf{R}^{\prime}$ for a SNP-effect biplot and $\mathbf{F F}$ ' = $\mathbf{C C}^{\prime}$ for a sample-effect biplot. For the convenience of visualization, a rank-2 approximate biplot is considered. Namely, the first two columns of $\mathbf{R}$ and $\mathbf{C}$ with the largest singular values are selected to approximate the allele frequency matrix F. A higher-rank approximate biplot is also plausible by visualizing more than two components pairwise.

\section{Appendix D. Estimation of the level of contamination by "normal" cells}

Individual-level allele frequency can also be used to calculate the level of contaminating "normal" cells. By extending the previous method [42], we derive a general formula to estimate the proportion of contamination by normal cells for tumor cell samples using individuallevel allele frequencies. Suppose that the study DNA is composed of $100(1-p) \%$ tumor cells and $100 p \%$ contaminating "normal" cells. In normal cells without AI, the ratio of two alleles of a heterozygous SNP is $1: 1$; in cancer cells with $\mathrm{AI}$, the ratio of two alleles is $a: b$, where $a$ and $b$ are the copies of the two alleles. Then the frequency of the first allele is $f_{1}=[p+a \times(1-p)] /\{[2-$ $(a+b)] \times p+(a+b)\}$ and the frequency of the second allele is $f_{2}=[(1-b) \times p+b] /\{[2-(a+b)] \times p+(a+b)\}$. Note that $p, a$, and $b$ cannot be zero simultaneously. Using simple algebra, the normal cell contamination proportion is derived to be $\left.p=\left[a \times\left(1-f_{1}\right)-b \times f_{1}\right] /\left[(1-b) \times f_{1}\right)-(1-a) \times f_{2}\right]$ if $(1-a) \times f_{2} \neq$ $(1-b) \times f_{1}$. As an example of a hemizygous deletion of the first allele (i.e., $a=0, b=1$ ), then $f_{1}=p /(1+p), f_{2}=1$ / $(1+p)$ and $p=f_{1} /\left(1-f_{1}\right)$. As an example of a single copy gain of the first allele (i.e., $a=2, b=1)$, then $f_{1}=(2-p)$ / $(3-p), f_{2}=1 /(3-p)$ and $p=\left(2-3 f_{1}\right) /\left(1-f_{1}\right)$.

\section{Additional material}

Additional file 1: Figure S1.-Allele frequency of an individual (NA18996) from the JPT population based on the Affymetrix Human Mapping 100K Set. This figure consists of 23 subfigures. Each subfigure presents an allele frequency plot of one chromosome. The vertical axis is the estimated allele frequency, and the horizontal axis is physical position (Mb). Each point denotes a SNP, and the gap in each subplot represents the centromeric gap. (A) Estimated allele frequency using an intensity-measuring approach. (B) Estimated allele frequency using an allele-counting approach.

Additional file 2: Figure S2-Unadjusted and adjusted individuallevel allele frequency of a sample (NA18940) from the JPT population based on the Affymetrix Human Mapping $100 \mathrm{~K}$ Set. This figure consists of 23 subfigures. Each subfigure presents an allele frequency plot of one chromosome. The vertical axis is the estimated allele frequency, and the horizontal axis is physical position (Mb). Each point denotes a SNP, and the gap in each subplot represents the centromeric gap. (A) Unadjusted individual-level allele frequency estimates. (B) CPA-adjusted individual-level allele frequency estimates.
Additional file 3: Figure S3.-Unadjusted and adjusted populationlevel allele frequency of an artificial DNA pool with a size of 240 individuals based on the Affymetrix Human Mapping $100 \mathrm{~K}$ Set. The vertical axis is the estimated allele frequency, and the horizontal axis is the true allele frequency. (A) Unadjusted population-level allele frequency estimates. (B) CPA-adjusted population-level allele frequency estimates.

Additional file 4: Figure S4.-Genomic distributions of CPA in $\log _{2}$ scale and standard error of CPA for four sample sizes. (A) This figure consists of 16 subfigures. The four diagonal subfigures are the histograms of $\log _{2}(\mathrm{CPA})$ for sample sizes of $367,180,90$ and 45 . The offdiagonal subfigures are scatter plots of $\log _{2}(C P A)$ for pairs of sample sizes, where each blue point denotes a $\log _{2}(C P A)$ value of a SNP. A quadratic regression curve is fitted. The mean regression curve is plotted in red, and the corresponding 95\% confidence interval is plotted in green. (B) The figure contains ratios of CPA standard errors of 180 versus 367 samples (green points), 90 versus 367 samples (red points) and 45 versus 367 samples (blue points). The red reference line denotes the ratio of 1, i.e., equal to the CPA standard error of 367 samples.

Additional file 5: Figure S5.-Genomic distributions of CPA in $\log _{2}$ scale for different data acquisition times (four genotyping periods) and experimental sites (two laboratories). (A) This figure consists of 16 subfigures. The four diagonal subfigures are the histograms of $\log _{2}$ (CPA) for the genotyping done on the time 2005/05/04, 2006/01/09, 2006/03/17 and 2006/06/29. The off-diagonal subfigures are scatter plots of $\log _{2}(C P A)$ for pairs of genotyping periods, where each blue point denotes a $\log _{2}(C P A)$ value of a SNP. (B) This figure consists of 23 subfigures. Each subfigure shows a scatter plot of CPAs in $\log _{2}$ scale of one chromosome based on 90 Asian samples in the HapMap project (vertical axis) and 95 Taiwanese samples (horizontal axis). A quadratic mean regression curve (red) and the corresponding 95\% confidence intervals (green) are calculated.

Additional file 6: Figure S6.-Genomic distributions of CPA in $\log _{2}$ scale based on the Affymetrix Human Mapping $100 \mathrm{~K}$ and $500 \mathrm{~K}$ Sets. This figure consists of 23 subfigures. Each subfigure shows a scatter plot or histogram of CPAs in $\log _{2}$ scale of one chromosome. (A) Scatter plots of CPAs in $\log _{2}$ scale for the Affymetrix Human Mapping 100K Set based on 457 Asian samples. (B) Histograms of CPAs in $\log _{2}$ scale for the Affymetrix Human Mapping 100K Set based on 457 Asian samples. (C) Scatter plots of CPAs in $\log _{2}$ scale for the Affymetrix Human Mapping 500K Set based on 538 Asian samples. (D) Histograms of CPAs in $\log _{2}$ scale for the Affymetrix Human Mapping 500K Set based on 538 Asian samples.

Additional file 7: Figure S7.-Lognormal distribution of CPA based on Taiwanese samples. CPAs are fitted using log-normal distributions by chromosome. The green curve is a fitted curve, and the purple curve is a theoretical lognormal curve. (A) Taiwanese samples (367 in total) that were genotyped with the Affymetrix Human Mapping 100K Set. (B) Taiwanese samples (448 in total) that were genotyped with the Affymetrix Human Mapping 500K Set.

Additional file 8: Figure S8.-Genomic distributions of CPA in $\log _{2}$ scale for different ethnic groups and a tree diagram. (A) This figure consists of 16 subfigures. The six diagonal subfigures are the histograms of $\log _{2}(\mathrm{CPA})$ for $45 \mathrm{CHB}, 45 \mathrm{JPT}, 60$ CEU founders, 60 YRI founders, 90 Asians (45 CHB and 45 JPT), and 210 combined samples. The off-diagonal subfigures are scatter plots of $\log _{2}(C P A)$ for pairs of groups, where each blue point denotes a $\log _{2}(C P A)$ value of a SNP. A quadratic mean regression curve (red) and the corresponding 95\% confidence intervals (green) are provided. (B) The studied populations are clustered according to between-population proximity (CPA correlation) via an average-linkage clustering analysis.

Additional file 9: Figure S9.-Genomic distributions of CPA in $\log _{2}$ scale for hypertensive case group and normotensive control group. This figure consists of 23 subfigures. Each subfigure shows a scatter plot of CPAs in $\log _{2}$ scale of one chromosome based on data from 175 hypertension patient samples (vertical axis) and 180 normal control samples (horizontal axis). A quadratic mean regression curve (red) and the corresponding 95\% confidence intervals (green) are shown. 
Additional file 10: Figure S10.-Allele frequency biplots of sex chromosomes of HapMap samples based on the Affymetrix Human Mapping 500K Set. (A) Allele frequency biplots of sex chromosomes for YRI populations in HapMap (30 fathers and 30 mothers in YRI). (B) Allele frequency biplots of sex chromosomes for CEU populations in HapMap (30 fathers and 30 mothers in CEU).

Additional file 11: Figure S11.-Allele frequency of a normal sample based on the Illumina HumanHap550-Duo BeadChip. This figure consists of 23 subfigures. Each subfigure presents an allele frequency plot of one chromosome. The vertical axis is the estimated allele frequency, and the horizontal axis is physical position (Mb). Each point denotes a SNP, and the gap in each subplot represents the centromeric gap. The allele frequencies were estimated using an intensity-measuring approach.

\section{Abbreviations}

Al: Allelic imbalance; ALL: Acute lymphoblastic leukaemia; ALOHA: Allelefrequency/Loss-of-heterozygosity/Allele-imbalance; CEU: CEPH Utah residents; CHB: Han Chinese in Beijing; CPA: Coefficient of preferential amplification/ hybridization; IGE: Individual genotyping experiment; JPT: Japanese in Tokyo; LCSH: Long contiguous stretch of homozygosity; LOH: Loss of heterozygosity; PAE: Pooled allelotyping experiment; SNP: Single nucleotide polymorphism; YRI: Yoruba in Ibadan.

\section{Acknowledgements}

We thank Ms Wen-Ting Fan for constructing CPA and allele frequency databases. We also thank Mr. Edward Cheng for his assistance with the Linux PC cluster system. We are grateful to Dr. Charles G. Mullighan for the provision of the acute lymphoblastic leukaemia project data. We gratefully acknowledge the National Clinical Core and National Genotyping Center at Academia Sinica for providing DNA samples and genotyping support. The work was supported by a grant from the National Science Council of Taiwan (NSC 97-2314-B-001-006-MY3) and the National Research Program for Genomic Medicine (NSC 97-3112-B-001-027 and NSC 97-3112B-001-013).

\section{Author details}

${ }^{1}$ Institute of Statistical Science, Academia Sinica, Taipei 115, Taiwan. ${ }^{2}$ Institute of Biomedical Sciences, Academia Sinica, Taipei 115, Taiwan.

\section{Authors' contributions}

HCY conceived of the study, developed statistical methods and prepared the manuscript. $\mathrm{HCL}$ and $\mathrm{MCH}$ programmed ALOHA software and analyzed the data with HCY. LHL contributed to discussion. WHP, JYW and YTC provided genetic data of Taiwanese samples. All authors read and approved the final manuscript.

\section{Received: 1 March 2010 Accepted: 5 July 2010 Published: 5 July 2010}

\section{References}

1. Cheung KH, Osier MV, Kidd JR, Pakstis AJ, Miller PL, Kidd KK: ALFRED: An allele frequency database for diverse populations and DNA polymorphisms. Nucleic Acids Research 2000, 28(1):361-363.

2. Sham P, Bader JS, Craig I, O'Donovan M, Owen M: DNA pooling: A tool for large-scale association studies. Nature Reviews Genetics 2002, 3(11):862-871.

3. Eberle MA, Rieder MJ, Kruglyak L, Nickerson DA: Allele frequency matching between SNPs reveals an excess of linkage disequilibrium in genic regions of the human genome. PLoS Genetics 2006, 2(9):e142.

4. Keinan A, Mullikin JC, Patterson N, Reich D: Measurement of the human allele frequency spectrum demonstrates greater genetic drift in East Asians than in Europeans. Nature Genetics 2007, 39:1251-1255.

5. Homer N, Szelinger S, Redman M, Duggan D, Tembe W, Muehling J, Pearson JV, Stephan DA, Nelson SF, Craig DW: Resolving individuals contributing trace amounts of DNA to highly complex mixtures using high-density SNP genotyping microarrays. PLOS Genetics 2008, 4(8): e1000167.
6. Yang HC, Huang MC, Li LH, Lin CH, Yu ALT, Diccianni MB, Wu JY, Chen YT, Fann CSJ: MPDA: Microarray pooled DNA analyzer. BMC Bioinformatics 2008, 9:196.

7. The International HapMap Consortium: The International HapMap Project. Nature 2003, 426(6968):789-796.

8. The International HapMap Consortium: Integrating ethics and science in the international HapMap project. Nature Reviews Genetics 2004, 5(6):467-475.

9. The International HapMap Consortium: A haplotype map of the human genome. Nature 2005, 437(7063):1299-1320.

10. The International HapMap Consortium: A second generation human haplotype map of over 3.1 million SNPs. Nature 2007, 449(7164):851-861.

11. Hoogendoorn B, Norton N, Kirov G, Williams N, Hamshere ML, Spurlock G, Austin J, Stephens MK, Buckland PR, Owen MJ, et al: Cheap, accurate and rapid allele frequency estimation of single nucleotide polymorphisms by primer extension and DHPLC in DNA pools. Human Genetics 2000, 107(5):488-493.

12. Yang HC, Pan CC, Lu RCY, Fann CSJ: New adjustment factors and sample size calculation in a DNA-pooling experiment with preferential amplification. Genetics 2005, 169(1):399-410.

13. Meaburn E, Butcher LM, Liu L, Fernandes C, Hansen V, Al-Chalabi A, Plomin R, Craig I, Schalkwyk LC: Genotyping DNA pools on microarrays: Tackling the QTL problem of large samples and large numbers of SNPs. BMC Genomics 2005, 6(1):52.

14. Moskvina V, Norton N, Williams N, Holmans P, Owen M, O'Donovan M: Streamlined analysis of pooled genotype data in SNP-based association studies. Genetic Epidemiology 2005, 28(3):273-282.

15. Simpson CL, Knight J, Butcher LM, Hansen VK, Meaburn E, Schalkwyk LC, Craig IW, Powell JF, Sham PC, Al-Chalabi A: A central resource for accurate allele frequency estimation from pooled DNA genotyped on DNA microarrays. Nucleic Acids Research 2005, 33(3):e25.

16. Bang-Ce Y, Zuo P, Yi BC, Li SY: Estimation of relative allele frequencies of single-nucleotide polymorphisms in different populations by microarray hybridization of pooled DNA. Analytical Biochemistry 2004, 333(1):72-78.

17. Yang HC, Liang YJ, Huang MC, Li LH, Lin CH, Wu JY, Chen YT, Fann CSJ: A genome-wide study of preferential amplification/hybridization in microarray-based pooled DNA experiments. Nucleic Acids Research 2006, 34(15):e106

18. Yin BC, Li HH, Ye BC: Microarray-based estimation of SNP allele-frequency in pooled DNA using the Langmuir kinetic model. BMC Genomics 2008, 9:605.

19. Docherty SJ, Butcher LM, Schalkwyk LC, Plomin R: Applicability of DNA pools on 500 KSNP microarrays for cost-effective initial screens in genomewide association studies. Bmc Genomics 2007, 8:214.

20. Pan WH, Fann CSJ, Wu JY, Hung YT, Ho MS, Tai TH, Chen YJ, Liao CJ, Yang ML, Cheng ATA, et al: Han Chinese cell and genome bank in Taiwan: Purpose, design and ethical considerations. Human Heredity 2006, 61(1):27-30.

21. Mullighan CG, Goorha S, Radtke I, Miller CB, Coustan-Smith E, Dalton JD, Girtman K, Mathew S, Ma J, Pounds SB, et al: Genome-wide analysis of genetic alterations in acute lymphoblastic leukaemia. Nature 2007, 446(7137):758-764.

22. Mullighan CG, Miller CB, Radtke I, Phillips LA, Dalton J, Ma J, White D, Hughes TP, Le Beau MM, Pui CH, et al: BCR-ABL1 lymphoblastic leukaemia is characterized by the deletion of Ikaros. Nature 2008, 453(7191):110-114.

23. Yang $H-C$, Liang $Y-J, W u ~ Y-L$, Chung $C-M$, Chiang $K-M$, Ho H-Y, Ting C-T, Lin T-H, Sheu S-H, Tsai W-C, et al: Genome-wide association study of young-onset hypertension in the Han Chinese population of Taiwan. PLOS ONE 2009, 4(5):e5459.

24. Di XJ, Matsuzaki H, Webster TA, Hubbell E, Liu GY, Dong SL, Bartell D, Huang J, Chiles R, Yang G, et al: Dynamic model based algorithms for screening and genotyping over 100K SNPs on oligonucleotide microarrays. Bioinformatics 2005, 21(9):1958-1963.

25. BRLMM: An improved genotype calling method for the GeneChip human mapping 500K array set. 2006.

26. Arnheim N, Strange C, Erlich H: Use of pooled DNA samples to detect linkage disequilibrium of polymorphic restriction fragments and human disease: Studies of the HLA class II loci. Proceedings of the National Academy of Sciences of the United States of America 1985, 82(20):6970-6974.

27. Butcher LM, Meaburn E, Liu L, Fernandes C, Hill L, Al-Chalabi A, Plomin R, Schalkwyk L, Craig IW: Genotyping pooled DNA on microarrays: $A$ 
systematic genome screen of thousands of SNPs in large samples to detect QTLs for complex traits. Behavior Genetics 2004, 34(5):549-555.

28. Pearson JV, Huentelman MJ, Halperin RF, Tembe WD, Melquist S, Homer N, Brun M, Szelinger S, Coon KD, Zismann VL, et al: Identification of the genetic basis for complex disorders by use of pooling-based genomewide single-nucleotide-polymorphism association studies. American Journal of Human Genetics 2007, 80(1):126-139.

29. Macgregor S, Zhao ZZ, Henders A, Martin NG, Montgomery GW, Visscher PM: Highly cost-efficient genome-wide association studies using DNA pools and dense SNP arrays. Nucleic Acids Research 2008, 36(6):e35.

30. Kirov G, Nikolov I, Georgieva L, Moskvina V, Owen MJ, O'Donovan MC: Pooled DNA genotyping on Affymetrix SNP genotyping arrays. BMC Genomics 2006, 7:27.

31. Yang H-C, Fann CSJ: Association mapping using pooled DNA. Methods in Molecular Biology Humana Press 2007, 376:161-175.

32. Huang T, Wu BL, Lizardi P, Zhao HY: Detection of DNA copy number alterations using penalized least squares regression. Bioinformatics 2005, 21(20):3811-3817.

33. Wang K, Li M, Hadley D, Liu R, Glessner J, Grant SF, Hakonarson H, Bucan M: PennCNV: an integrated hidden Markov model designed for highresolution copy number variation detection in whole-genome SNP genotyping data. Genome Research 2007, 17(11):1665-1674.

34. Colella S, Yau C, Taylor JM, Mirza G, Butler H, Clouston P, Bassett AS, Seller A, Holmes CC, Ragoussis J: QuantiSNP: An objective Bayes HiddenMarkov Model to detect and accurately map copy number variation using SNP genotyping data. Nucleic Acids Research 2007, 35(6):2013-2025.

35. Li C, Beroukhim R, Weir BA, Winckler W, Garraway LA, Sellers WR, Meyerson M: Major copy proportion analysis of tumor samples using SNP arrays. BMC Bioinformatics 2008, 9:204.

36. Sun W, Wright FA, Tang ZZ, Nordgard SH, Van Loo P, Yu TW, Kristensen VN, Perou CM: Integrated study of copy number states and genotype calls using high-density SNP arrays. Nucleic Acids Research 2009, 37(16):5365-5377.

37. Heckman NE, Ramsay JO: Penalized regression with model-based penalties. Canadian Journal of Statistics 2000, 28(2):241-258.

38. Gabriel KR: The biplot graphic display of matrices with application to principal component analysis. Biometrika 1971, 58(3):453-467.

39. Chapman S, Schenk P, Kazan K, Manners J: Using biplots to interpret gene expression patterns in plants. Bioinformatics 2002, 18(1):202-204.

40. Pittelkow YE, Wilson SR: Visualisation of gene expression data - the GEbiplot, the Chip-plot and the Gene-plot. Stat Appl in Genet and Mol Biol 2003, 2:Article6.

41. Huggins R, Li LH, Lin YC, Yu AL, Yang HC: Nonparametric estimation of LOH using Affymetrix SNP genotyping arrays for unpaired samples. J Hum Genet 2008, 53(11-12):983-990.

42. Staaf J, Lindgren D, Vallon-Christersson J, Isaksson A, Goransson $H_{\text {, }}$ Juliusson G, Rosenquist R, Hoglund M, Borg A, Ringner M: Segmentationbased detection of allelic imbalance and loss-of-heterozygosity in cancer cells using whole genome SNP arrays. Genome Biology 2008, 9(9).

doi:10.1186/1471-2164-11-415

Cite this article as: Yang et al:: A new analysis tool for individual-level

allele frequency for genomic studies. BMC Genomics 2010 11:415.

\section{Submit your next manuscript to BioMed Central and take full advantage of:}

- Convenient online submission

- Thorough peer review

- No space constraints or color figure charges

- Immediate publication on acceptance

- Inclusion in PubMed, CAS, Scopus and Google Scholar

- Research which is freely available for redistribution

Submit your manuscript at www.biomedcentral.com/submit 